\title{
DISERTACIONES
}

AVANCES

Para citar este artículo: Boos de Quadros, C. M., Durieux Zucco, F., Foletto Fiuza, T., \& De Souza Farias, F. (2021). Fuentes de información, credibilidad y publicidad: perspectivas para el desarrollo de la comunicación regional. Anuario Electrónico de Estudios en Comunicación Social "Disertaciones", 14(1), 1-20. https:// doi.org/10.12804/revistas.urosario.edu.co/disertaciones/a.9003

\section{FUENTES DE INFORMACIÓN, CREDIBILIDAD Y PUBLICIDAD: PERSPECTIVAS PARA EL DESARROLLO DE LA COMUNICACIÓN REGIONAL}

\section{Sources of Information, Credibility, and Advertising: Perspectives for the Development of Regional Communication}

\section{Fontes de informação, credibilidade e publicidade: perspectivas para o desenvolvimento da comunicação regional}

Cynthia Morgana Boos de Quadros, Universidade Regional de Blumenau (Santa Catarina, Brasil)

cynthia@furb.br

Fabricia Durieux Zucco, Universidade do Vale do Itajaí (Balneário Camboriú, Santa Catarina, Brasil). Universidade Regional de Blumenau (Santa Catarina, Brasil) fabricia.zucco@gmail.com

Thamires Foletto Fiuza, Universidade do Vale do Itajaí (Balneário Camboriú, Santa Catarina, Brasil)

thamiresf.fiuza@gmail.com

Fernanda de Souza Farias, Universidade do Vale do Itajaí (Balneário Camboriú, Santa Catarina, Brasil)

nandasfarias@hotmail.com 
Recibido: 29 de abril de 2020

Aceptado: 10 de septiembre de 2020

Fecha de prepublicación:

\title{
RESUMEN
}

Este artículo analiza las fuentes de información sobre la vida cotidiana de los habitantes de Blumenau (Santa Catarina, Brasil) y su credibilidad. Por medio de un análisis exploratorio y descriptivo con perspectiva cuantitativa, el estudio de encuesta se centró en la identificación de las fuentes de información más utilizadas y en el análisis de la credibilidad atribuida a los medios de comunicación y los vehículos más usados para los receptores de información, es decir, aquellos que investigan contenidos en fuentes de información. La investigación demostró que internet y medios de comunicación tradicionales (TV, radio y periódicos) son la fuente principal de información de temas cotidianos para los blumenauenses y, al mismo tiempo, gozan de mayor credibilidad. Tales resultados proporcionan pistas sobre oportunidades y perspectivas para el desarrollo de la publicidad regional.

Palabras clave: fuentes de información; credibilidad; publicidad; Blumenau.

\begin{abstract}
In this research article, sources of information on the daily life of the inhabitants of Blumenau (Santa Catarina, Brazil) and their credibility were analyzed. Through an explorative and descriptive analysis with a quantitative approach, the survey-based study focused on identifying the most frequently used information sources and analyzing the credibility of the communication media and the most frequently used vehicles by information recipients, that is, those that search for content in sources of information. The research proved that the Internet and conventional communication media (TV, radio, and newspapers) are the primary sources of information for topics related to daily life among the people in Blumenau, and at the same time, they have greater credibility. Such results provide hints regarding opportunities and perspectives for the development of regional advertising.
\end{abstract}

Keywords: Sources of information; credibility; advertising; Blumenau.

\section{RESUMO}

Este artigo analisa as fontes de informação sobre o cotidiano dos moradores de Blumenau (Santa Catarina, Brasil) e sua credibilidade. Por meio de uma análise exploratória e descritiva com abordagem quantitativa, o estudo baseado em pesquisa se concentrou na identificação das fontes de informação mais utilizadas e na análise da credibilidade atribuída à mídia e aos veículos mais utilizados pelos destinatários da informação, isto é, aqueles que pesquisam conteúdo em fontes de informação. A pesquisa mostrou que a Internet e as mídias tradicionais 


\section{DISERTACIONES}

AVANCES

Los desafios del periodismo narrativo

ISSN: $1856-9536$

Doi: https://doi.org/10.12804/revistas.urosario.edu.co/disertaciones/a.9003

Volumen 14, Número 1 / Enero-junio 2021

Versión PDF para imprimir desde

http://revistas.urosario.edu.co/index.php/disertaciones

(TV, rádio e jornais) são as principais fontes sobre o cotidiano dos Blumenauenses e, ao mesmo tempo, possuem maior credibilidade. Tais resultados fornecem pistas sobre oportunidades e perspectivas para o desenvolvimento da publicidade regional.

Palavras-chave: fontes de informação; credibilidade; publicidade; Blumenau.

En las últimas décadas, los estudios relacionados con la búsqueda de información han ganado importancia en la investigación académica, adoptando diferentes perspectivas de pesquisa y dando lugar a una serie de modelos teóricos y métodos para la evaluación del comportamiento del consumidor (Maity et al., 2014; Mortimer \& Pressey, 2013; Utkarsh \& Medhavi, 2015). De hecho, las personas buscan y usan información constantemente como parte de su vida diaria. Informaciones relacionadas con el trabajo, el ocio, la salud, el dinero, el clima, la familia, productos y servicios son puestas a disposición desde múltiples fuentes. Los investigadores han prestado una atención sustancial a este asunto debido a su primacía en el comportamiento de búsqueda, así como en la toma de decisiones del consumidor (Utkarsh \& Medhavi, 2015).

Según Dinet et al. (2012), la búsqueda de información es un proceso dinámico, en el cual las herramientas utilizadas varían de acuerdo con el tiempo, el usuario y las necesidades bajo las cuales ocurre. Esta perspectiva se basa en la percepción de una serie de trabajos conforme con los cuales es esencial conocer al usuario de la información, entender su comportamiento en la búsqueda y también identificar qué fuentes/canales de información utiliza más (Maity et al., 2014; Mortimer \& Pressey, 2013; Parratt Fernández, 2010). Los expertos argumentan también que la confianza y credibilidad, tanto de las informaciones como de los informadores, condicionan el proceso de búsqueda y la selección informativa de los usuarios (Arias Robles, 2014).

El campo de la comunicación continúa evolucionando rápidamente a medida que la tecnología digital cambia la naturaleza de la información y cómo las personas interactúan y aprenden de/con la información emitida desde la web (Dinet et al., 2012). Al referirse a la llamada "sociedad de la información", Castells (2002) argumenta que esta se caracteriza no porque encaje en un modelo específico de estructura social, sino porque organiza su sistema de producción en torno a principios de maximización de la productividad basados en el conocimiento, a través del desarrollo y la difusión de tecnologías de la información. Las redes sociales se constituyen en un instrumento generalizado de consumo y producción de información. Con su creciente disponibilidad, se han convertido en herramientas universales para compartir y consumir información en una variedad de formatos de contenido.

En este contexto, el presente trabajo busca analizar las fuentes de información sobre la vida cotidiana de Blumenau (Santa Catarina, Brasil) y relacionarlas con la credibilidad de los medios y vehículos de comunicación. Por medio de un análisis exploratorio y descriptivo con perspectiva cuantitativa, aplicado a los habitantes de la ciudad, 


\section{DISERTACIONES}

\section{AVANCES}

Los desafios del periodismo narrativo

ISSN: 1856-9536

Doi: https://doi.org/10.12804/revistas.urosario.edu.co/disertaciones/a.9003

Volumen 14, Número 1 / Enero-junio 2021

Versión PDF para imprimir desde

http://revistas.urosario.edu.co/index.php/disertaciones

quienes se caracterizan como receptores de información, el estudio se orienta a la consecución de los siguientes objetivos: 1) identificar las fuentes de información más utilizadas en la busca de informaciones sobre la vida cotidiana en Blumenau; 2) relacionar los vehículos de comunicación con las características de la población; 3) analizar la credibilidad atribuida a los medios de comunicación y los vehículos más utilizados por personas de Blumenau; y 4) presentar oportunidades y perspectivas para el desarrollo de la publicidad regional. Con base en los resultados obtenidos, se muestran las conclusiones más relevantes y direcciones futuras en este campo de estudio.

\section{Marco teórico}

\section{Fuentes de información}

Definir fuentes de información no es tarea simple. En muchas ocasiones, la principal dificultad para dar una explicación esclarecedora de lo que son o de lo que se entiende por fuentes de información se debe a que este concepto se define sobre la base de la tipología de fuentes que existen. El término fomenta diversas reflexiones académicas y ha evolucionado con el tiempo, generando nuevas perspectivas y enfoques. Según Villaseñor (1998), inicialmente se asociaba con las bibliografías y las obras de referencia. Definiciones actuales, más amplias y generalistas, consideran como fuentes de información todos los instrumentos y recursos que sirven para satisfacer las necesidades informativas de cualquier persona, y que contienen información general o especializada, independientemente del soporte (Cordón García, 1998; Villaseñor, 1998).

Si bien no existe una tipología unificada respecto a las fuentes de información, es posible encontrar criterios clasificatorios que sirven para conocer la amplia gama de recursos informativos en que se han constituido estas fuentes. Algunos de ellos son: 1) por la procedencia y origen de la información: fuentes personales, institucionales, documentales; 2) por el canal utilizado para transmitir la información: de transmisión oral, documental, digital; ${ }^{1}$ 3) por la cobertura geográfica: internacional, nacional, regional, local; 4) por el grado de adecuación de la información que ofrecen: total, media, insuficiente; 5) por el tipo de información que presentan: especializada o general; 6) por el nivel informativo/contenido: fuentes primarias, secundarias, terciarias (Chatterjee, 2017; Martín Vega, 1995; Villaseñor, 1998). Estos criterios se complementan, de forma que puede darse el hecho de que una misma fuente de información sea, por ejemplo, de procedencia institucional, de transmisión digital, con cobertura geográfica internacional y de carácter especializado.

Con los avances tecnológicos y el uso generalizado de internet, la cantidad de fuentes de información disponibles ha aumentado significativamente (Xiang, 2018). Además de las fuentes tradicionales de información, han surgido otras como resultado de una mayor conectividad, aplicaciones móviles y redes sociales (García-Milon et al., 2020). Esta 'nueva realidad' hizo que en las últimas décadas diversas disciplinas se preocuparan por comprender cómo las personas buscan y utilizan la información, cuáles son las fuentes y los canales que emplean, así como los factores que inhiben o fomentan el comportamiento de búsqueda de información (Wilson, 2000; Xiang, 2018). Sobre este asunto se parte del criterio de que los individuos, después de reconocer el problema (falta de información), inician un proceso de búsqueda para solucionarlo de la mejor manera posible. Para autores como Mowen y

1 Según Chatterjee (2017), esta tipología puede ser clasificada como tangible (fuentes impresas) o intangible (fuentes digitales). 


\section{DISERTACIONES}

AVANCES

Los desafios del periodismo narrativo

ISSN: $1856-9536$

Doi: https://doi.org/10.12804/revistas.urosario.edu.co/disertaciones/a.9003

Volumen 14, Número 1 / Enero-junio 2021

Versión PDF para imprimir desde

http://revistas.urosario.edu.co/index.php/disertaciones

Minor (2003), Solomon (2016) y García-Milon et al. (2020), este aspecto puede desarrollarse de dos maneras posibles: por medio de una búsqueda de informaciones internas o teniendo en cuenta fuentes de información externa.

Las fuentes de información interna se absorben mediante diversas sensaciones que provienen de respuestas inmediatas a los receptores sensoriales (ojos, oídos, nariz, boca, piel). También se absorben a través de la percepción, que es el proceso que selecciona, interpreta y organiza estas sensaciones (Solomon, 2016). De esa forma, la búsqueda interna se presenta como un proceso de recordar información, sentimientos y experiencias almacenados en la memoria, y que a menudo se pueden recuperar (García-Milon et al., 2020). Hoyer y Maclnnis (2012) señalan que la búsqueda de información interna se centra muchas veces en lo que se rescata, que pueden ser marcas, atributos, evaluaciones y experiencias.

La búsqueda de información con fuentes externas se utiliza para obtener un conocimiento adicional del que ya se tiene, y para identificar nuevas oportunidades, atributos y beneficios asociados en el conjunto de consideraciones (García-Milon et al., 2020; Hoter \& Maclnnis, 2012). Para Solomon (2016), el consumidor realiza una investigación externa cuando es un tema importante o cuando es fácil obtener información relevante sobre el asunto. Por otra parte, Blackwell et al. (2005) expresan que este tipo de búsqueda no se limita a fuentes dominadas por algún área de actuación, sino que incorpora otras, como profesionales del mercado, amigos, familiares, líderes de opinión, medios y actualmente también a través de youtubers e influencers digitales. A medida que el consumidor se expone a la información a través de búsquedas externas, comienza a procesar estímulos que incluyen:

1. Exposición: primero, la información persuasiva y la comunicación deben llegar a los consumidores.

2. Atención: después de la exposición, el siguiente paso es asignar (o no asignar) la capacidad de procesamiento de información para la información entrante. Cuanto más relevante sea el contenido del mensaje, más atención atraerá. 3. Comprensión: si se atrajo la atención, el mensaje se analizará de acuerdo con las categorías de significado almacenadas en la memoria. 4. Aceptación: una vez que se lleva a cabo la comprensión, el mensaje puede considerarse inaceptable (un resultado común) y, por lo tanto, dispensado o aceptado. El propósito del mensaje es modificar o alterar las creencias o actitudes existentes, pero el mensaje debe ser aceptado antes de que esto suceda. 5. Retención: finalmente, el objetivo de quienes persuaden es que la nueva información sea aceptada y almacenada en la memoria de manera accesible para su uso futuro (Blackwell et al., 2005, p. 90).

De manera general, las personas buscan información que les ayude a comprender mejor situaciones cotidianas para poder tomar decisiones al respecto. Atendiendo a ese criterio, Savolainen (2006) desarrolló un modelo conceptual que enfatiza "el [...] papel de los factores sociales y culturales que afectan el estilo de las personas en la preferencia y el uso de las fuentes de información en situaciones cotidianas" (p. 143). Para este autor, el everyday life information seeking (ELIS) $)^{2}$ se caracteriza como un intento de abordar el fenómeno de buscar información para la vida cotidiana, combinando factores sociales y psicológicos. La figura 1 presenta el modelo eus. Su punto de partida es el estilo de vida, que permite la investigación de factores individuales y sociales. Se puede argumentar entonces que las actividades de orientación y resolución de problemas involucran tanto la búsqueda de información como la toma de decisiones diarias.

2 Búsqueda de información en el ámbito de la vida cotidiana (traducción autoral en español). 


\section{DISERTACIONES}

AVANCES

\section{COTIDIANO}

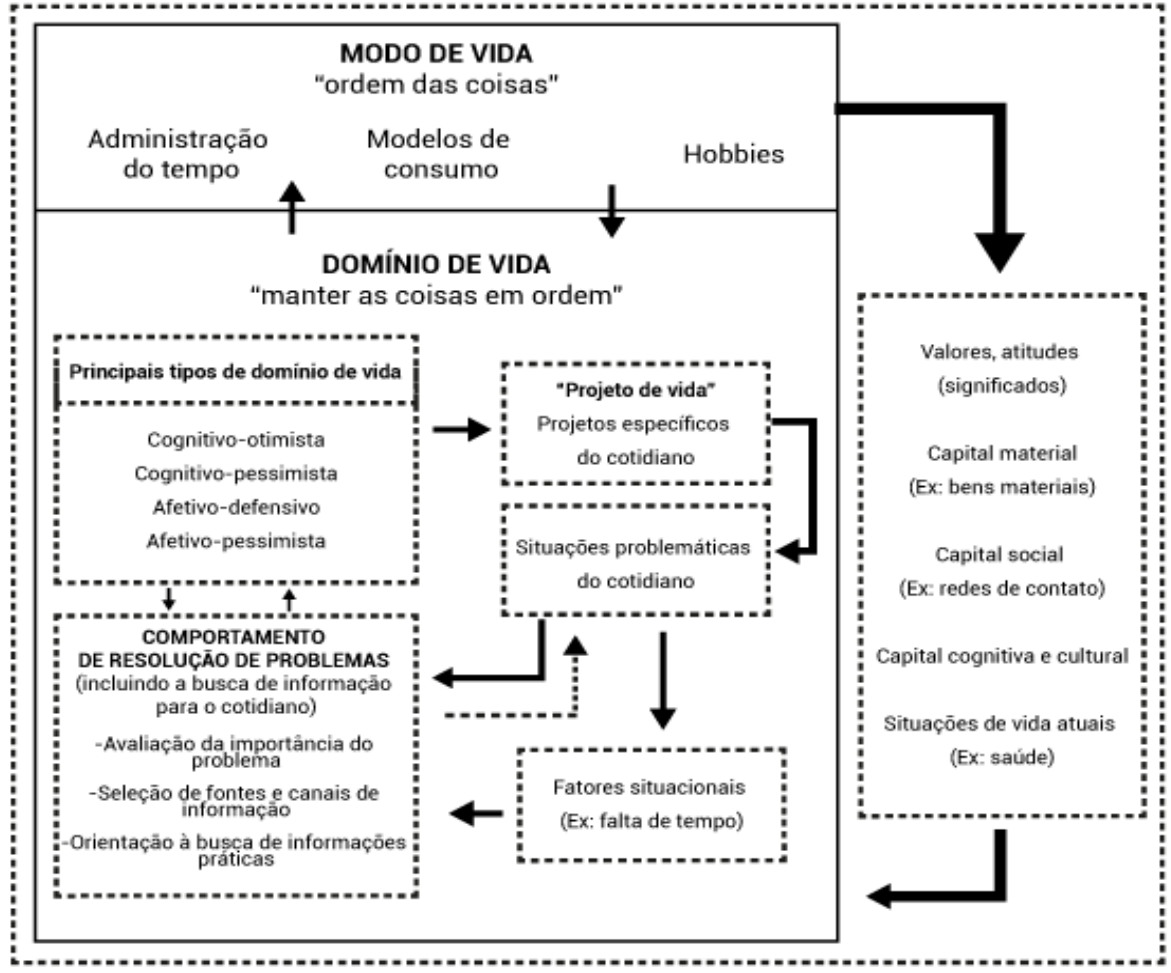

Figura 1. Modelo de búsqueda de información en el ámbito de la vida cotidiana

Fuente: adaptado de Savolainen (2006). Traducido al portugués por Rocha et al. (2017).

Como se muestra en el modelo anterior, los dominios de la vida están influenciados por el comportamiento de resolución de problemas de los sujetos y también influyen en ellos. Estas influencias se deben a tres factores principales: la evaluación de la importancia del problema, la selección de fuentes y canales de información, y la orientación en la búsqueda de información práctica. Los elementos que guían la búsqueda de información dentro del dominio de la vida pueden citarse como factores situacionales, situaciones problemáticas de la vida cotidiana y proyectos de vida. A partir de este modelo, otros investigadores realizaron diferentes estudios refiriéndose a la búsqueda de información en la vida cotidiana, a menudo adaptando el modelo a las situaciones actuales (Agosto \& Hassel, 2005; Carey et al., 2001; Sin \& Kim, 2013; Williamson et al., 2013; Loudon et al., 2016).

\section{Credibilidad de los medios de comunicación}

Los medios de comunicación de masas median las relaciones sociales entre diferentes grupos e influyen fuertemente en la organización de la sociedad, actuando como formadores de la opinión pública e instrumentos de intervención en la vida social (Funk \& McCombs, 2017; Brum, 2003; Guareschi \& Biz, 2005). Para Castells (2002), los medios funcionan como un sistema integrado en el cual los periódicos informan el evento y preparan análisis, la 


\section{DISERTACIONES}

\section{AVANCES}

Los desafios del periodismo narrativo

ISSN: $1856-9536$

Doi: https://doi.org/10.12804/revistas.urosario.edu.co/disertaciones/a.9003

Volumen 14, Número 1 / Enero-junio 2021

Versión PDF para imprimir desde

http://revistas.urosario.edu.co/index.php/disertaciones

televisión lo digiere y lo difunde al público en general, y la radio ofrece la oportunidad de participación ciudadana. En ese conjunto, se incluye la internet, que está transformando los contornos de la vida diaria, confundiendo los límites entre lo global y lo local, y presentando -al mismo tiempo- nuevos canales de comunicación e interacción (Giddens, 2005), especialmente entre la población más joven.

Thompson (2012) argumenta que las personas están involucradas en un proceso de formación personal y autocomprensión al recibir y apropiarse de los mensajes provenientes de los medios. De acuerdo con Sordi et al. (2008), la credibilidad de las fuentes información se convierte en un problema creciente, especialmente con las nuevas herramientas digitales y plataformas mediáticas. Tal fenómeno, según Metzger et al. (2003), consta de dos dimensiones principales: la especialización y la confiabilidad de los medios. La especialización se refiere a la evaluación individual de la capacidad del comunicador para hacer declaraciones correctas, mientras que la confiabilidad se refiere al grado en que el individuo se da cuenta de que el comunicador está diciendo la verdad. De esa forma, las fuentes que exhiban tales dimensiones disfrutan de ventajas significativas sobre sus competidores menos confiables, cuando se trata de influencia social.

La confiabilidad es importante para una comunicación eficaz porque la disposición de las personas para aceptar mensajes comunicativos está relacionada con el grado en que confían en la información y su fuente (Mackeracher et al., 2018). Por lo tanto, la confiabilidad en una fuente puede influir en cómo fluye la información a través de los sistemas sociales y en el nivel de influencia que puede alcanzar (Chen \& Cheng, 2019; Ismagilova et al., 2020). La confiabilidad se puede lograr de varias maneras, pero Metzger et al. (2003) argumentan que la especialización y la riqueza de los detalles verificados son los principales aspectos que influyen. Así, Ismagilova et al. (2020) sostienen que la fuente de información y las recomendaciones brindadas se consideran confiables si la declaración se cree válida, honesta y objetiva, y esto genera confianza en la fuente de la información.

Cuando se habla de confianza en los medios de comunicación, se entiende como la actitud, creencia o expectativa favorable de un sujeto hacia un canal informativo (Mackeracher et al., 2018). Por otro lado, al hacer referencia a la credibilidad se apuntan los atributos característicos del medio, los cuales contribuyen a que la audiencia deposite o no su confianza en él. Se pueden mencionar la difusión de información verídica, la marca, la transparencia, la reputación, la imparcialidad, la tendencia hacia el manejo informativo del interés público, contar con colaboradores reconocidos y de prestigio, y difundir mensajes de calidad que promuevan la convivencia pacífica, la libertad, la justicia y la democracia, entre otros valores (Denegri et al., 2015).

Ismagilova et al. (2020) afirman que, cuando un consumidor piensa que la información proporcionada es de una fuente altamente confiable, percibirá la información como útil. Investigaciones anteriores, como la de Visentin et al. (2019), encontraron que la confiabilidad de la fuente afecta la utilidad percibida de las comunicaciones. Así, el aumento de la confianza en una fuente de información puede ayudar a comprender en quién confían las personas para obtener información, facilitando el compromiso y las estrategias de comunicación específicas diseñadas para incrementar la credibilidad de una fuente (Visentin et al., 2019).

La credibilidad de la noticia es un elemento fundamental para la comunicación pública, que a su vez es esencial para que una democracia más saludable funcione en una sociedad (Lee et al., 2020). Transmitir noticias de manera imparcial es la clave para generar credibilidad y confianza en la información, sin embargo, el desafío es operacionalizar lo imparcial (Lee, 2018). 


\section{DISERTACIONES}

\section{AVANCES}

Los desafios del periodismo narrativo

ISSN: 1856-9536

Doi: https://doi.org/10.12804/revistas.urosario.edu.co/disertaciones/a.9003

Volumen 14, Número 1 / Enero-junio 2021

Versión PDF para imprimir desde

http://revistas.urosario.edu.co/index.php/disertaciones

Para verificar cómo se construye la credibilidad informativa en los anuncios de los periódicos españoles, se analizaron los periódicos impresos entre 1618 y 1635 (Soto-Escobar \& Espejo-Cala, 2019). Se constató que, a pesar de la apelación constante a la credibilidad de los textos, en términos generales, no había interés en poner a disposición las fuentes de los datos publicados en los periódicos españoles, dejando al lector la búsqueda de más información sobre las fuentes (Soto-Escobar \& Espejo-Cala, 2019).

Lee (2018), por su parte, encontró que los estadounidenses parecen tener una confianza baja o en declive en los medios de comunicación. Bennett y Livingston (2018) atribuyen la difusión de información falsa a la falta de legitimidad de una sociedad, ya que, con la caída de la confianza ciudadana en algunas instituciones informativas, la credibilidad de la información en los medios oficiales disminuye y abre espacio para la búsqueda de fuentes alternativas de información.

En el caso de Brasil, conforme con la Encuesta de Medios Brasileños realizada en 2016 con 15050 personas, la fuente de información de mayor credibilidad es el periódico, con casi un $60 \%$. Este dato se confirma en un estudio global más reciente Trust in the media, del instituto Ipsos, el cual indica que la credibilidad de periódicos y revistas brasileras es la cuarta mayor del mundo ( 6 de cada 10 brasileros confían en esos vehículos). La radio (un $29 \%$ siempre confía y el $28 \%$ a menudo) y la televisión (un 27,5\% siempre y el 25,5\% a menudo) también son medios de gran credibilidad. Por el contrario, según la encuesta de 2016, los blogs, redes sociales y también sitios de internet son los medios de comunicación con menor credibilidad en Brasil. Esto se debe, en cierta forma, por el fuerte impacto de la desinformación difundida en las redes sociales en los últimos años (Ipsos, 2019).

No es nuevo que cuestionen la credibilidad de los medios, tanto es así que este es un tema ampliamente discutido por los investigadores en la actualidad (Lee, 2018; Han, 2018; Berhanu \& Raj, 2020). Un ejemplo de ello es la cuestión de la credibilidad de la información como valor democrático, que ha sido un aspecto de esta temática y un asunto de gran interés para los investigadores de la última década (Lee et al., 2020).

Una de las preocupaciones relacionadas con la credibilidad en los medios es la cuestión de la falsedad de la información. Los medios de comunicación, con el fin de movilizar a los partidarios de la prensa que transmitan sus mensajes, han experimentado niveles crecientes de difusión de información falsa, especialmente en lo que respecta a cuestiones políticas (Bennett \& Livingston, 2018).

En el estudio desarrollado por Yamamoto y Nah (2018), los autores buscan probar si la credibilidad percibida de los periódicos locales se explica por factores a nivel individual y comunitario. A nivel individual, los autores confirmaron la proposición de que los individuos tienden a usar una determinada fuente de información porque confían en ella, confirmando los estudios de Lee (2018), que dice que es común consumir fuentes de noticias que se creen confiables.

Berhanu y Raj (2020) desarrollaron un estudio con el objetivo de abordar la confiabilidad de las fuentes de información sobre viajes y turismo de las plataformas de redes sociales. Este estudio se llevó a cabo con 310 visitantes, quienes tuvieron una percepción positiva sobre la confiabilidad de la información de viajes en las redes sociales. Los visitantes con la percepción más favorable de la confiabilidad de la información tenían entre 18 y 35 años. A medida que aumenta el grupo de edad, la percepción de confiabilidad se vuelve menos favorable (Berhanu \& Raj, 2020). 


\section{DISERTACIONES}

\section{AVANCES}

Los desafios del periodismo narrativo

ISSN: 1856-9536

Doi: https://doi.org/10.12804/revistas.urosario.edu.co/disertaciones/a.9003

Volumen 14, Número 1 / Enero-junio 2021

Versión PDF para imprimir desde

http://revistas.urosario.edu.co/index.php/disertaciones

\section{Búsqueda de información, toma de decisión y publicidad}

De acuerdo con Kulviwat et al. (2004), un consumidor inmerso en un proceso de compra puede sentir la necesidad de contar con una determinada cantidad de información que le sirva fundamentalmente para aminorar los riesgos relacionados con sus posteriores decisiones. En el entorno global dinámico de hoy, comprender cómo las audiencias buscan y usan la información, y cómo esta influye en el proceso de consumo y de toma de decisiones es crucial para diseñar campañas efectivas de comunicación publicitaria.

Los teóricos de la toma de decisiones reconocen el papel de la información en este proceso (Radford, 1994) y lo dividen en cinco pasos: reconocimiento de problemas, búsqueda de información, evaluación de alternativas, compra y evaluación posterior a la compra (Lamb et al., 2002). La presente investigación se centra en la búsqueda de información y cómo este paso facilita las actividades de decisión de compra. De esta forma, el origen y calidad de la información que necesitan las audiencias en su proceso de búsqueda es un factor importante por tener en cuenta en toda estrategia de comunicación publicitaria.

En términos de exposición, las fuentes relacionadas con el ámbito comercial (líderes de opinión, influencers, cuentas/perfiles institucionales, vendedores, vallas publicitarias, enlaces patrocinados, etc.) son muy importantes, pues son los medios y/o vehículos seleccionados específicamente para el mensaje publicitario. No obstante, fuentes supuestamente neutras (periódicos, revistas, radio, Tv, etc.), que eliminan de cierta forma el carácter mercantilista de la información, son más efectivas y creíbles de cara al consumidor. En este sentido, resulta primordial identificar los medios de comunicación que ofrezcan una mayor probabilidad de respuestas favorables a la publicidad (Zeugner-Roth et al., 2015).

Considerando la difusión de internet y las redes sociales, los consumidores tienen acceso a más fuentes que facilitan su necesidad informativa y los ayudan a tomar decisiones (Park \& Kim, 2003). Sin embargo, la cuestión importante por tomar en consideración relacionada con la búsqueda es conocer si realmente el número de alternativas valoradas por el consumidor antes de tomar la decisión también se ve incrementado. Además, la lealtad hacia la marca dependerá en gran medida de la satisfacción y credibilidad de la información recibida y el beneficio relacional (Park \& Kim, 2003). En consecuencia, es necesario que las empresas inviertan en tecnología de tal forma que puedan adaptar sus servicios a las expectativas y necesidades de los clientes.

\section{Metodología}

El objetivo general de esta investigación es analizar las fuentes de información sobre la vida cotidiana de Blumenau (Santa Catarina, Brasil) percibidas por los receptores de la información, relacionar los vehículos de comunicación con las características de la población y verificar la confianza de los blumenauenses en cuanto a las fuentes de información aquí analizadas. Atendiendo a ese propósito, se buscó realizar un levantamiento bibliográfico donde se sistematizaron las principales temáticas que sirvieron como base para la elaboración del marco teórico-conceptual al cual se acoge la presente investigación.

Posteriormente, se aplicó una investigación descriptiva, con enfoque cuantitativo, donde se elaboró el instrumento de recolección de datos y se aplicó con los blumenauenses. Conforme con Diehl (2004), esta metodología utiliza procedimientos cuantitativos tanto en la recopilación de datos como en el tratamiento de la información 


\section{DISERTACIONES}

AVANCES

Los desafios del periodismo narrativo

ISSN: 1856-9536

Doi: https://doi.org/10.12804/revistas.urosario.edu.co/disertaciones/a.9003

Volumen 14, Número 1 / Enero-junio 2021

Versión PDF para imprimir desde

http://revistas.urosario.edu.co/index.php/disertaciones

obtenida, usando técnicas estadísticas que eviten posibles distorsiones de análisis e interpretación, lo que permite un menor margen de error y una mayor seguridad de la investigación.

El estudio empírico empleó como instrumento de recopilación un cuestionario semiestructurado, compuesto por preguntas cerradas y aplicado a sujetos de interés, con el fin de obtener datos generales y conocer sus percepciones acerca del tema de estudio. Tal instrumento fue elaborado con base en los siguientes extractos teórico-metodológicos: búsqueda de información en la vida cotidiana (basada en el modelo ELIs), creado por Savolainen (2006), específicamente la relación entre el trabajo y el ocio, los modelos de consumo y la naturaleza de los pasatiempos. Además, se tuvieron en cuenta los factores del individuo, así como sus características y la situación actual de su vida. La dimensión de credibilidad de los medios de comunicación tuvo como base teórica los estudios desarrollados por Savolainen (2006), Christofoletti y Laux (2008) y Hoyer y Maclnnis (2012).

Fueron considerados como población de este estudio los 352460 habitantes de la ciudad de Blumenau, según lo estimado por el IBGE en 2018. La muestra, de tipo no probabilístico, fue compuesta por 400 residentes, con edad superior a 16 años, que acostumbran buscar información sobre asuntos de la vida cotidiana. Entre los temas cuestionados pueden citarse: salud y bienestar, economía, noticias de la ciudad, política brasilera, moda, belleza y estilo de vida, turismo, entretenimiento y ocio, trabajo, productos y servicios, clima y previsión del tiempo, deporte, alimentación y gastronomía.

Los datos fueron recopilados por un equipo de investigación, integrantes del proyecto Focus de la Universidad Regional de Blumenau. El $100 \%$ de los cuestionarios completados fueron validados. Aquellos incompletos, tachados o sin contacto telefónico para el procedimiento de verificación fueron descartados de la muestra, y el $30 \%$ de los cuestionarios fueron sometidos a verificaciones telefónicas para garantizar la veracidad de los datos. De esa forma, la muestra final fue de 210 sujetos, que llevan un margen de error del 6,8\%, para un nivel de confianza del $95 \%$ en relación con la población de Blumenau. Para aplicar la verificación de confiabilidad y consistencia interna de las variables, se utilizó el software Microsoft Excel para la codificación y tabulación de datos. Posteriormente, para el tratamiento y análisis de estos, se usó el paquete estadístico Statistical Package for Social Science (sPss).

\section{Resultados y discusión}

A continuación, son presentados los resultados obtenidos al aplicar las técnicas de investigación seleccionadas. Para la caracterización sociodemográfica de los sujetos de pesquisa, se consideraron datos personales como sexo, edad, escolaridad e ingresos. La información sobre la descripción de la muestra se puede ver en la tabla 1. Como es una encuesta con preguntas de opción múltiple, el número total de opciones de respuesta puede ser mayor que el número de encuestados que respondieron la pregunta, por lo tanto, esto puede causar que los porcentajes totales de respuestas superen el $100 \%$. 


\section{DISERTACIONES}

\section{AVANCES}

Los desafios del periodismo narrativo

ISSN: 1856-9536

Doi: https://doi.org/10.12804/revistas.urosario.edu.co/disertaciones/a.9003

Volumen 14, Número 1 / Enero-junio 2021

Versión PDF para imprimir desde

http://revistas.urosario.edu.co/index.php/disertaciones

Tabla 1. Caracterización sociodemográfica de los sujetos de pesquisa

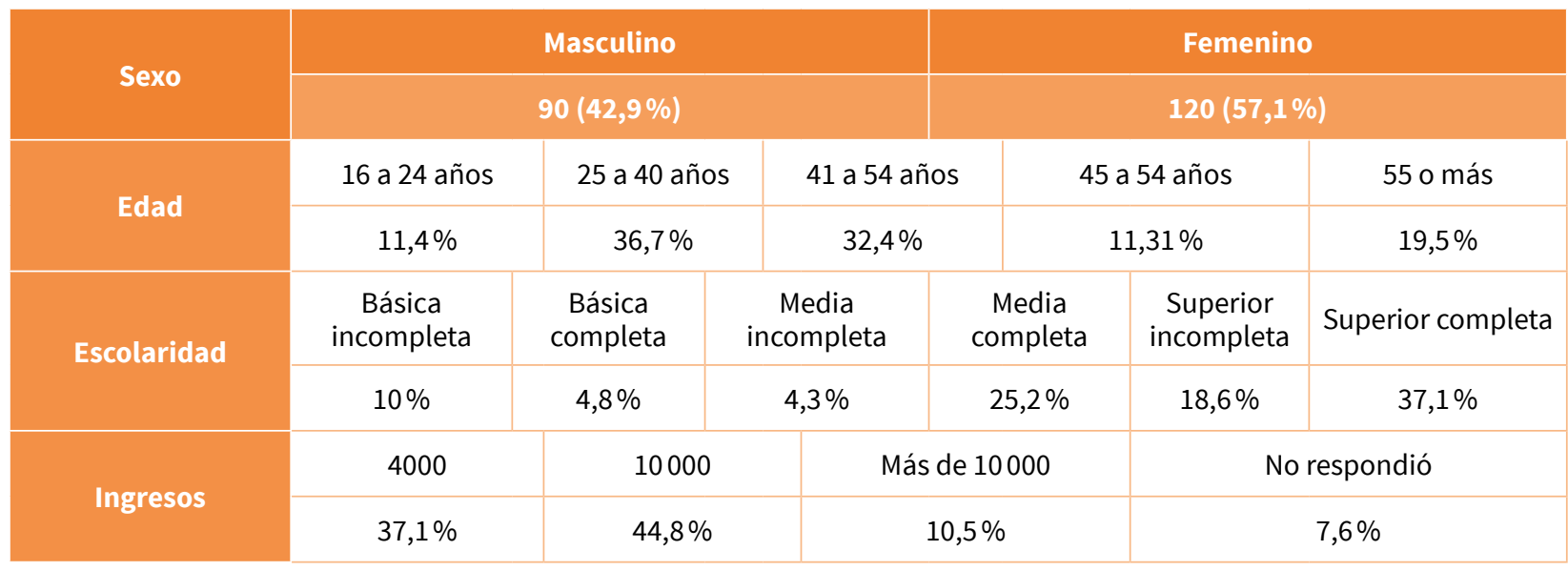

Fuente: elaboración de los autores.

Los encuestados eran en su mayoría mujeres $(57,1 \%)$. Con respecto al grupo de edad, se puede decir que el público es diverso, la mayor parte de las respuestas corresponde a las categorías de jóvenes y adultos, con edades comprendidas entre 25 y 54 años $(69,1 \%)$. La caracterización del público en términos de escolaridad nos permite observar que los encuestados tienen un nivel alto de educación, con enseñanza superior completa/incompleta con un 55,7\%. En términos de ingresos, se percibe que el público posee ingresos totales significativos (55,3\%).

\section{Fuentes de información y su credibilidad}

Siguiendo con el análisis, la tabla 2 presenta las fuentes de información más utilizadas por los blumenauenses para buscar informaciones de la vida cotidiana. Con una media de 3,52 fuentes señaladas por cada sujeto (pregunta de opción múltiple), los principales valores corresponden a internet (69\%), televisión (50\%), periódicos $(40,5 \%)$; seguido de amigos (31,9\%), radio $(26,7 \%)$ y Facebook $(24,8 \%)$.

Tabla 2. Fuentes de información más utilizadas

\begin{tabular}{|c|c|c|}
\hline Fuente & Frecuencia & $\%$ \\
\hline Internet & 145 & 69,0 \\
\hline TV & 105 & 50,0 \\
\hline Periódicos & 85 & 40,5 \\
\hline Amigos & 67 & 31,9 \\
\hline Radio & 56 & 26,7 \\
\hline
\end{tabular}




\begin{tabular}{|c|c|c|}
\hline Fuente & Frecuencia & $\%$ \\
\hline Facebook & 52 & 24,8 \\
\hline Familiares & 46 & 21,9 \\
\hline Instagram & 36 & 17,1 \\
\hline WhatsApp & 30 & 14,3 \\
\hline Profesionales del área & 26 & 12,4 \\
\hline Influenciadores digitales & 23 & 11,0 \\
\hline Revistas & 21 & 10,0 \\
\hline Formadores de opinión & 13 & 6,2 \\
\hline Películas y videos & 12 & 5,7 \\
\hline Literatura & 8 & 3,8 \\
\hline Profesores & 7 & 3,3 \\
\hline Twitter & 4 & 1,9 \\
\hline Celebridades & 2 & 1,0 \\
\hline Comerciantes & 2 & 1,0 \\
\hline
\end{tabular}

Fuente: elaboración de los autores.

Tales resultados muestran que, a pesar de que medios de comunicación tradicionales (periódicos, tv y radio) constituyen aún fuentes de información cardinales, las nuevas tecnologías (internet y redes sociales) han ganado un espacio destacado en la preferencia de las audiencias para informarse sobre la vida cotidiana en Blumenau. Esos recursos tecnológicos ofrecen un acceso versátil y posibilitan una mayor interacción con el contenido. En el caso específico de la internet, se percibe que los encuestados lo refieren como el lugar donde están disponibles todo tipo de servicios e información, de ahí su frecuencia significativa en las respuestas.

Posteriormente, con el propósito de identificar la relación entre las variables de investigación se efectuó un cruce (tabla 3) entre las variables sexo, edad e ingresos con las fuentes de información. 


\section{DISERTACIONES}

AVANCES

Los desafios del periodismo narrativo

ISSN: 1856-9536

Doi: https://doi.org/10.12804/revistas.urosario.edu.co/disertaciones/a.9003 Volumen 14, Número 1 / Enero-junio 2021

Versión PDF para imprimir desde

http://revistas.urosario.edu.co/index.php/disertaciones

Tabla 3. Cruce de variables categóricas (\%)

\begin{tabular}{|c|c|c|c|c|c|c|c|c|c|c|}
\hline \multirow{2}{*}{$\begin{array}{l}\text { Fuentes de } \\
\text { información }\end{array}$} & \multicolumn{2}{|c|}{ Sexo } & \multicolumn{4}{|c|}{ Edad } & \multicolumn{4}{|c|}{ Ingresos } \\
\hline & M & $\mathbf{F}$ & $16 / 24$ & $25 / 40$ & $41 / 54$ & $55 /+$ & $\begin{array}{c}\text { Hasta } \\
4000\end{array}$ & $\begin{array}{l}\text { Hasta } \\
10000\end{array}$ & $\begin{array}{l}\text { Más de } \\
10000\end{array}$ & $\begin{array}{l}\text { No } \\
\text { respondió }\end{array}$ \\
\hline Familiares & 26,6 & 18,3 & 37,5 & 23,4 & 25,0 & 4,9 & 14,1 & 28,7 & 18,2 & 25,0 \\
\hline Amigos & 35,5 & 29,1 & 50,0 & 33,8 & 27,9 & 24,4 & 21,8 & 38,3 & 45,5 & 25,0 \\
\hline Profesores & 6,6 & 0,8 & 12,5 & 2,6 & 2,9 & 0,0 & 1,3 & 4,3 & 4,5 & 6,3 \\
\hline $\begin{array}{l}\text { Formadores de } \\
\text { opinión }\end{array}$ & 7,7 & 5,0 & 4,4 & 7,8 & 8,8 & 0,0 & 5,1 & 8,5 & 0,0 & 0,0 \\
\hline Celebridades & 0,0 & 1,6 & 0,0 & 1,3 & 1,5 & 0,0 & 1,3 & 1,1 & 0,0 & 6,3 \\
\hline $\begin{array}{l}\text { Influenciadores } \\
\text { digitales }\end{array}$ & 15,5 & 7,5 & 20,8 & 11,7 & 10,3 & 4,9 & 7,7 & 8,5 & 36,4 & 6,3 \\
\hline $\begin{array}{l}\text { Profesionales del } \\
\text { área }\end{array}$ & 12,2 & 12,5 & 8,3 & 18,2 & 10,3 & 7,3 & 6,4 & 17,0 & 18,2 & 0,0 \\
\hline Comerciantes & 1,1 & 0,0 & 0,0 & 0,0 & 2,9 & 0,0 & 1,3 & 1,1 & 0,0 & 43,8 \\
\hline Periódicos & 41,1 & 40,0 & 58,3 & 35,1 & 39,7 & 41,5 & 38,5 & 41,5 & 40,9 & 25,0 \\
\hline Revistas & 12,2 & 8,3 & 4,2 & 10,4 & 8,8 & 14,6 & 11,5 & 7,4 & 4,5 & 56,3 \\
\hline TV & 48,8 & 50,8 & 37,5 & 39,0 & 57,4 & 65,9 & 59,0 & 41,5 & 50,0 & 18,8 \\
\hline Radio & 35,5 & 20,0 & 33,3 & 20,8 & 27,9 & 31,7 & 24,4 & 27,7 & 36,4 & 56,3 \\
\hline Internet & 70,0 & 68,3 & 79,2 & 85,7 & 64,7 & 39,9 & 56,4 & 79,8 & 77,3 & 56,3 \\
\hline Facebook & 27,7 & 22,5 & 25,0 & 32,5 & 20,6 & 17,1 & 11,5 & 33,0 & 31,8 & 31,3 \\
\hline Instagram & 14,4 & 19,1 & 16,7 & 32,5 & 8,8 & 2,4 & 9,0 & 22,3 & 31,8 & 6,3 \\
\hline WhatsApp & 16,6 & 12,5 & 12,5 & 15,6 & 13,2 & 14,6 & 15,4 & 13,8 & 18,2 & 6,3 \\
\hline Twitter & 3,3 & 0,8 & 0,0 & 2,6 & 2,9 & 0,0 & 0,0 & 4,3 & 0,0 & 0,0 \\
\hline Filmes y videos & 6,6 & 5,0 & 12,5 & 7,8 & 4,4 & 0,0 & 3,8 & 6,4 & 9,1 & 6,3 \\
\hline Literatura & 3,3 & 4,1 & 0,0 & 3,9 & 4,4 & 4,9 & 5,1 & 4,3 & 0,0 & 0,0 \\
\hline
\end{tabular}

Fuente: elaboración de los autores. 


\section{DISERTACIONES}

\section{AVANCES}

De acuerdo con los datos obtenidos, se confirma que la internet es la fuente de información principal para todos los grupos de edades e ingresos. De igual forma, el periódico y la tv reflejan un alto índice de frecuencia para todas las edades (entre el $35 \%$ y $65,9 \%$ en cada grupo etario, y entre el $38 \%$ y $59 \%$ en cada grupo de ingresos generados). Sobre el público joven (adolescente y adulto joven), Meštrović et al. (2019) argumentan que las informaciones y experiencias que se derivan de relaciones con iguales sirven de modelo y referencia para la toma de decisiones de la vida diaria; en ese sentido, los datos resultantes permitieron observar que dicho público (16/24 años $=50 \% ; 25 / 40$ años $=33,8 \%$ ) manifiesta un interés de utilizar como fuentes de información a los amigos.

En el caso de la radio, se encontró que el público masculino joven (33\%) y aquel de más de 55 años (31\%) emplean con frecuencia la radio como fuente de información para las noticias locales y los temas de la vida cotidiana. Tales cifras confirman los resultados del estudio de Kantar IBOPE en $2019^{3}$ que indicó que la radio llega al $83 \%$ de los brasileros y el mayor promedio de uso está entre las personas más jóvenes (19-30 años, 86\%) y adultos mayores (más de 50, 90\%). Es preciso resaltar además que redes sociales como Facebook e Instagram constituyen fuentes de información importantes para el público comprendido entre 25 y 40 años (32,5\%) y para aquellos con más de 10000 reales de ingreso (31,8\%).

Por otra parte, y de acuerdo con los datos obtenidos en la investigación, fue posible recopilar informaciones sobre la credibilidad de los medios de comunicación para los blumenauenses, siendo utilizada una escala de confianza. Fueron seleccionados los medios de comunicación con mayor frecuencia de uso.

Tabla 4. Confianza en los medios de comunicación

\begin{tabular}{l|c|c|c|c|c|c|c} 
& No confío & Confío poco & Indiferente & $\begin{array}{c}\text { Confío } \\
\text { razonablemente }\end{array}$ & $\begin{array}{c}\text { Confío } \\
\text { mucho }\end{array}$ & No sé & Total \\
\hline TV & $\%$ & $\%$ & $\%$ & $\%$ & $\%$ & $\%$ & $\%$ \\
\hline Radio & 12,4 & 35,2 & 6,2 & 39,0 & 6,7 & 0,5 & 100,0 \\
\hline Revista & 5,7 & 23,8 & 10,5 & 37,6 & 13,8 & 8,6 & 100,0 \\
\hline Periódico & 13,3 & 28,6 & 9,5 & 32,4 & 7,1 & 9,0 & 100,0 \\
\hline Facebook & 5,2 & 15,7 & 11,9 & 43,8 & 12,9 & 10,5 & 100,0 \\
\hline Instagram & 40,5 & 28,6 & 6,7 & 11,9 & 0,5 & 11,9 & 100,0 \\
\hline WhatsApp & 28,6 & 20,5 & 8,1 & 12,4 & 1,0 & 29,5 & 100,0 \\
\hline
\end{tabular}

Fuente: elaboración de los autores.

3 Kantar ibope Media 2019. Rádio alcança $83 \%$ dos brasileiros e é mais popular entre os jovens. https:// www.kantaribopemedia.com/radio-alcanca-83-dos-brasileiros-e-e-mais-popular-entre-os-jovens/ 


\section{DISERTACIONES}

\section{AVANCES}

Los desafios del periodismo narrativo

ISSN: $1856-9536$

Doi: https://doi.org/10.12804/revistas.urosario.edu.co/disertaciones/a.9003

Volumen 14, Número 1 / Enero-junio 2021

Versión PDF para imprimir desde

http://revistas.urosario.edu.co/index.php/disertaciones

Conforme con los resultados, los mayores porcentajes de confianza se encuentran en el periódico, siendo que el $43,8 \%$ confíarazonablemente y el $12,9 \%$ confía mucho; seguido de la radio donde un $37,6 \%$ confía razonablemente y un 13,8\% confía mucho. En tercer lugar de credibilidad está la televisión, con el $39 \%$ de confianza razonable y el $6,7 \%$ de mucha confianza. Esto hace posible afirmar que los blumenausenses que buscan informaciones sobre la vida cotidiana tienen mayor confianza en los medios tradicionales (periódico, radio y Tv).

En contrapartida, redes sociales como Facebook (40,5\%), Instagram (28,6\%) y WhatsApp (41\%) tienen altos índices de desconfianza, lo que se traduce en una percepción de fuentes con baja credibilidad. Estos medios, al mismo tiempo que ofrecen un mayor acceso a informaciones de la vida cotidiana y a más versiones de eventos, reflejan ciertas inconsistencias y brechas en la cobertura de noticias con diferentes interpretaciones del mismo evento o informaciones incompletas o falsas. Esto hace que el público termine sintiéndose engañado, lo que conlleva una erosión en la credibilidad de estos medios de comunicación.

\section{Oportunidades y perspectivas para el desarrollo de la publicidad regional}

La búsqueda de información de un consumidor se vuelve compleja cuando hay una gran cantidad de alternativas disponibles en el mercado. Esta variable puede desempeñar un papel más importante en el dominio de internet, donde con un solo clic los consumidores tienen una gran cantidad de opciones. Los datos obtenidos son importantes cuando se tienen en cuenta los factores que influyen en la elección del consumidor para ciertos productos y servicios en los procesos de decisión de compra. Varios estudios muestran que la búsqueda de información y la capacidad de persuadir al público preceden al consumo de un producto o servicio (Mowen \& Minor, 2003; Sheth et al., 2001). En este proceso, la credibilidad de la fuente, que involucra la experiencia percibida, adquiere relevancia debido a la confianza que las personas tienen en las opiniones que estas emiten (Turcotte et al., 2015).

La credibilidad es un tema constantemente discutido por el Consejo Nacional de Autorregulación Publicitaria (Conar). Gilberto Carlos Leifert, expresidente de la entidad, enfatiza que la publicidad depende de la credibilidad, pues un anuncio honesto y verdadero, de acuerdo con las leyes y la ética, satisface las necesidades de credibilidad de la industria y la necesidad del público de recibir información correcta y útil (Rodrigues, 2010). De esa forma, mientras más informadas estén las personas, mejor podrán tomar decisiones.

Toda esta dinámica impone un desafío para las empresas de cualquier sector (Wolton, 2006). Para el ámbito publicitario, el desafío es doble. Por una parte, implica conocer las actitudes y percepciones de los destinatarios del mensaje sobre determinado producto/servicio. Por otra, implica identificar el medio de comunicación adecuado para desarrollar y aplicar estrategias comunicativas consistentes con las más diversas audiencias. En este contexto, los profesionales de la comunicación de Blumenau deben ser conscientes de la percepción del público sobre los medios utilizados para transmitir publicidad, ya que la forma en que se percibe tiene un impacto significativo en la manera en que se reciben y absorben los mensajes publicitarios, visión compartida por Fraser y McBain (2011).

Consecuentemente, muchas organizaciones blumenauenses invierten grandes sumas en los medios de comunicación, buscando asegurar el contacto entre su mensaje y sus consumidores, lo que aumenta los costos de una campaña. Por lo tanto, la integración competente de los medios seleccionados por los anunciantes se convierte en un factor crítico de éxito para una campaña (Briggs et al., 2005). Si a esto le sumamos el uso y dependencia del 


\section{DISERTACIONES}

AVANCES

Los desafios del periodismo narrativo

ISSN: $1856-9536$

Doi: https://doi.org/10.12804/revistas.urosario.edu.co/disertaciones/a.9003

Volumen 14, Número 1 / Enero-junio 2021

Versión PDF para imprimir desde

http://revistas.urosario.edu.co/index.php/disertaciones

público a las nuevas tecnologías de información y comunicación para realizar las tareas diarias, se percibe cómo este fenómeno ha impactado directamente en la forma en que los anunciantes crean, planean e implementan la publicidad (Machado Filho \& Lima, 2017). Para los autores, los medios tradicionales y nuevos son complementarios, y si se usan juntos, ofrecen una gama de posibilidades para anunciantes, productores y vehículos de comunicación (Machado Filho \& Lima, 2017).

En el caso de Blumenau, los resultados de la investigación demuestran que los medios tradicionales dominan aspectos relacionados con el uso para informaciones de la vida cotidiana y constituyen los medios más confiables y creíbles para las audiencias. De igual forma, se percibe que internet y algunas redes sociales vienen ganando espacio e influencia en estos asuntos. En este sentido, resulta esencial observar el escenario que se presenta para el desarrollo de la publicidad regional. Se precisa crear alternativas que consigan influir sobre las decisiones de consumo de las personas e integren efectivamente los medios tradicionales y digitales. Esta convergencia requiere una ruptura con el modelo tradicional de comunicación con los consumidores, obligando al sector publicitario blumenauense a buscar opciones creativas que despierten interés en el contenido publicitario vehiculado en aquellos medios de comunicación de preferencia local.

\section{Conclusiones}

El objetivo central de este trabajo se enfocó en analizar las fuentes de información sobre la vida cotidiana de Blumenau (Santa Catarina, Brasil) y relacionarlas con la credibilidad de los medios y vehículos de comunicación. De esa forma, fue realizado un análisis exploratorio y descriptivo con perspectiva cuantitativa para identificar las fuentes de información más utilizadas en la busca de informaciones sobre la vida cotidiana en Blumenau; analizar la credibilidad atribuida a los medios de comunicación y los vehículos más utilizados por personas de Blumenau; y, finalmente, presentar oportunidades y perspectivas para el desarrollo de la publicidad regional.

En términos generales se puede concluir que internet y los medios de comunicación tradicionales (TV, radio y periódicos) son la fuente principal de información de temas cotidianos para los blumenauenses. De acuerdo con los resultados, al buscar informaciones estos depositan mayor confianza en los medios tradicionales (periódico, radio y TV), pues constituyen los medios más creíbles para la mayoría de los encuestados. Las redes sociales como Facebook, Instagram, WhatsApp, en contrapartida, presentaron altos índices de desconfianza, lo que se traduce en una percepción de fuentes con baja credibilidad.

Aunque los resultados del presente estudio se refieren a un contexto socioeconómico, geográfico y cultural particular, se cree que pueden extenderse a otros mercados con características similares. A partir de los resultados de este estudio se sugiere que trabajos futuros se centren en el conocimiento de las actividades que constituyen la búsqueda de información emprendida por los consumidores antes de la compra, pues es fundamental para capacitar a la empresa en el diseño eficaz de estrategias de comunicación acordes a las pautas de comportamiento de los consumidores. 


\section{DISERTACIONES}

AVANCES

Los desafíos del periodismo narrativo

ISSN: 1856-9536

Doi: https://doi.org/10.12804/revistas.urosario.edu.co/disertaciones/a.9003

Volumen 14, Número 1 / Enero-junio 2021

Versión PDF para imprimir desde

http://revistas.urosario.edu.co/index.php/disertaciones

\section{Referencias}

1. Agosto, D. E., \& Hassel, S. H. (2005). People, places, and questions: an investigation of the everyday life information-seeking behaviors of urban young adults. Library \& Information Science Research, 27(2), 141-163.

2. Arias Robles, F. (2014). La credibilidad de los contenidos informativos en internet para los 'nativos digitales': estudio de caso. Palabra Clave, 17(3), 875-894.

3. Bennett, W. L., \& Livingston, S. (2018). The disinformation order: disruptive communication and the decline of democratic institutions. European Journal of Communication, 33(2), 122-139.

4. Berhanu, K., \& Raj, S. (2020). The trustworthiness of travel and tourism information sources of social media: perspectives of international tourists visiting Ethiopia. Heliyon, 6(3), e03439.

5. Blackwell, R. D., Engel, J. F., \& Miniard, P. W. (2005). Comportamento do consumidor. Pioneira Thomson Learning.

6. Briggs, R., Krishnan, R., \& Borin, N. (2005). Integrated multichannel communication strategies: the case of the 2004 Ford F-150 launch. Journal of Interactive Marketing, 19(3), 81-90.

7. Brum, J. (2003). A hipótese do agenda setting: estudos e perspectivas. Razón y Palabra, 35. http://www. razonypalabra.org.mx/anteriores/n35/jbrum.html

8. Carey, R. F., McKechnie, L., \& McKenzie, P. J. (2001). Gaining access to everyday life information seeking. Library \& Information Science Research, 23(4), 319-334.

9. Castells, M. (2002). A era da informação. Economia, sociedade e cultura. Vol. I. A sociedade em rede. Fundação Calouste Gulbenkian.

10. Chatterjee, A. (2017). Elements of information organization and dissemination. Chandos Publishing.

11. Chen, Z. F., \& Cheng, Y. (2019). Consumer response to fake news about brands on social media: the effects of self-efficacy, media trust, and persuasion knowledge on brand trust. Journal of Product \& Brand Management, 29(2), 188-198.

12. Christofoletti, R., \& Laux, A. (2008). Confiabilidade, credibilidade e reputação: no jornalismo e na blogosfera. Intercom-Revista Brasileira de Ciências da Comunicação, 31(1), 29-49.

13. Cordón García, J. A. (1998). Las fuentes de información hoy. Criterios de selección y evaluación. En I. de Torres Ramírez (Coord.), Las fuentes de información: estudios teórico-prácticos (pp. 43-62). Síntesis.

14. Denegri, C., et al. (2015). Creencias y actitudes hacia los medios de comunicación en estudiantes universitarios del sur de Chile. Signo y Pensamiento, 34(67), 112-129.

15. Diehl, A. (2004). Pesquisa em ciências sociais aplicadas: métodos e técnicas. Pearson Education.

16. Dinet, J., Chevalier, A., \& Tricot, A. (2012). Information search activity: an overview. Revue Européenne de Psychologie Appliquée, 62(2), 49-62.

17. Fraser, K., \& McBain, J. (2011). Advertising: what the uk really thinks. Credos. http://www. adassoc.org.uk/ wp-content/uploads/2014/09/Advertising-What-the-UK-really-thinks.pdf

18. Funk, M. J., \& McCombs, M. (2017). Strangers on a theoretical train: inter-media agenda setting, community structure, and local news coverage. Journalism Studies, 18(7), 845-865. 


\section{DISERTACIONES}

AVANCES

Los desafios del periodismo narrativo

ISSN: 1856-9536

Doi: https://doi.org/10.12804/revistas.urosario.edu.co/disertaciones/a.9003

Volumen 14, Número 1 / Enero-junio 2021

Versión PDF para imprimir desde

http://revistas.urosario.edu.co/index.php/disertaciones

19. García-Milon, A., Juaneda-Ayensa, E., Olarte-Pascual, C., \& Pelegrín-Borondo, J. (2020). Towards the smart tourism destination: key factors in information source use on the tourist shopping journey. Tourism Management Perspectives, 36, 100730.

20. Giddens, A. (2005). Sociología (4a ed.). Artmed.

21. Guareschi, P. A., \& Biz, O. (2005). Mídia, educação e cidadania. Vozes.

22. Han, K. (2018). How do you perceive this author? Understanding and modeling authors' communication quality in social media. PLOS ONE, 13(2), 58-96.

23. Hoyer, W. D., \& Maclnnis, D. J. (2012). Comportamento do consumidor. Cengage Learning.

24. Ipsos. (2019). Ipsos Global Advisor. Trust in the media. https://www.ipsos.com/sites/default/files/ct/news/ documents/2019-06/global-advisor-trust-in-media-2019.pdf

25. Ismagilova, E., Slade, E., Rana, N. P., \& Dwivedi, Y. K. (2020). The effect of characteristics of source credibility on consumer behaviour: a meta-analysis. Journal of Retailing and Consumer Services, 53, 1-9.

26. Kantar ibope Media. (2019). Rádio alcança $83 \%$ dos brasileiros e é mais popular entre os jovens. https:// www.kantaribopemedia.com/radio-alcanca-83-dos-brasileiros-e-e-mais-popular-entre-os-jovens/

27. Kulviwat, S., Guo, C., \& Engchanil, N. (2004). Determinants of on-line information search: a critical review and assessment. Internet Research, 14(3), 245-253.

28. Lamb, C., Hair, H., \& McDaniel, C. (2002). Marketing (6 $6^{\mathrm{a}}$ ed.). Thomson Learning.

29. Lee, S., Nah, S., Chung, D. S., \& Kim, J. (2020). Predicting al news credibility: communicative or social capital or both? Communication Studies, 71(3), 428-447.

30. Lee, T.-T. (2018). Virtual theme collection: "Trust and credibility in news media". Journalism \& Mass Communication Quarterly, 95(1), 23-27.

31. Loudon, K., Buchanan, S., \& Ruthven, I. (2016). The everyday life information seeking behaviours of firsttime mothers. Journal of Documentation, 72(1), 24-46.

32. Machado Filho, F., \& Lima, C. E. (2017). Os desafios da publicidade na tv na era dos dados e da convergência. Revista da Set, 26(171). https://set.org.br/news-revista-da-set/revista/os-desafios-dapublicidade-na-tv-na-era-dos-dados-e-da-convergencia/

33. MacKeracher, T., Diedrich, A., Gurney, G. G., \& Marshall, N. (2018). Who trusts whom in the Great Barrier Reef? Exploring trust and communication in natural resource management. Environmental Science \& Policy, 88, 24-31.

34. Maity, M., Dass, M., \& Malhotra, N. K. (2014). The antecedents and moderators of offline information search: a meta-analysis. Journal of Retailing, 90(2), 233-254.

35. Martín Vega, A. (1995). Fuentes de información general. Trea.

36. Meštrović, D., et al. (2019). Information sources and factors influencing enrolment in ict and Stem University study programmes. Economic and Business Review, 21(1), 37-56.

37. Metzger, M., et al. (2003). Bringing the concept of credibility into the $21^{\text {st }}$ century: integrating perspectives on source, message, and media credibility in the contemporary media environment. Communication Yearbook, 27, 293-335.

38. Mortimer, K., \& Pressey, A. (2013). Consumer information search and credence services: implications for service providers. Journal of Services Marketing, 27(1), 49-58. 


\section{DISERTACIONES}

AVANCES

Los desafíos del periodismo narrativo

ISSN: 1856-9536

Doi: https://doi.org/10.12804/revistas.urosario.edu.co/disertaciones/a.9003

Volumen 14, Número 1 / Enero-junio 2021

Versión PDF para imprimir desde

http://revistas.urosario.edu.co/index.php/disertaciones

39. Mowen, J. C., \& Minor, S. (2003). Comportamento do consumidor. Prentice Hall.

40. Park, C., \& Kim, Y. (2003). Identifying key factors affecting consumer purchase behavior in an online shopping context. International Journal of Retail \& Distribution Management, 31(1), 16-29.

41. Parratt Fernández, S. (2010). Consumo de medios de comunicación y actitudes hacia la prensa por parte de los universitarios. ZER, 15(28), 133-149.

42. Radford, K. J. (1994). Decision making, individuals. En V. S. Ramachandran (Ed.), Encyclopedia of human behavior (pp. 73-82). Academic Press.

43. Rocha, J., Duarte, A., \& Paula, C. (2017). Modelos de práticas informacionais. Questão, 23(1), 36-61.

44. Rodrigues, L. (2010). Presidente do Conar critica atitude da Anvisa de regular propaganda de alimentos. O Globo. https://oglobo.globo.com/economia/presidente-do-conar-critica-atitude-da-anvisa-de-regular-propaganda-de-alimentos-2973499

45. Savolainen, R. (2006). Everyday life information seeking. En K. Fischer et al. (Eds.), Theories of information behavior (pp. 143-148). Information Today Inc.

46. Sheth, J. N., Mittal, B., \& Newman, B. I. (2001). Comportamento do cliente: indo além do comportamento do consumidor. Atlas.

47. Sin, S., \& Kim, K. (2013). International students' everyday life information seeking. The informational value of social networking sites. Library \& Information Science Research, 35(2), 107-116.

48. Solomon, M. R. (2016). Comportamento do consumidor: comprando, possuindo e sendo (11 ed.). Bookman.

49. Sordi, J. O., Meireles, M., \& Grijo, R. (2008). Gestão da qualidade da informação no contexto das organizações: percepções a partir do experimento de análise da confiabilidade dos jornais eletrônicos. Perspectivas em Ciência da Informação, 13(2), 168-195.

50. Soto-Escobar, R., \& Espejo-Cala, C. (2019). The information sources of the first Spanish newspapers: the construction of information credibility. Communication \& Society, 32(3), 81-92.

51. Thompson, J. B. (2012). A mídia e a modernidade: uma teoria social da mídia (13 ed.). Vozes.

52. Turcotte, J., et al. (2015). News recommendations from social media opinion leaders: effects on media trust and information seeking. Journal of Computer Mediated Communication, 20(5), 520-535.

53. Utkarsh, D., \& Medhavi, S. (2015). Information search behaviour of service consumers. The Marketing Review, 15(2), 201-219.

54. Villaseñor, I. (1998). Los instrumentos para la recuperación de información: las fuentes. En I. de Torres Ramírez (Coord.), Las fuentes de información: estudios teórico-prácticos (pp. 29-42). Síntesis.

55. Visentin, M., Pizzi, G., \& Pichierri, M. (2019). Fake news, real problems for brands: the impact of content truthfulness and source credibility on consumers' behavioral intentions toward the advertised brands. Journal of Interactive Marketing, 45, 99-112.

56. Williamson, K., et al. (2013). Young adults and everyday-life information: the role of news media. Library \& Information Science Research, 34(4), 258-264.

57. Wilson, T. D. (2000). Human information behavior. Special Issue on Information Science Research, 3(2), 49-55.

58. Wolton, D. (2006). É preciso salvar a comunicação. Paulus. 


\section{DISERTACIONES}

AVANCES

59. Xiang, Z. (2018). From digitization to the age of acceleration: on information technology and tourism. Tourism Management Perspectives, 25, 147-150.

60. Yamamoto, M., \& Nah, S. (2018). A multilevel examination of local newspaper credibility. Journalism \& Mass Communication Quarterly, 95(1), 76-95.

61. Zeugner-Roth, K., et al. (2015). Consumer ethnocentrism, national identity, and consumer cosmopolitanism as drivers of consumer behavior: a social identity theory perspective. Journal of International Marketing, 23(2), 25-54. 